\title{
The plasmacytoid dendritic cell: at the cross-roads in asthma
}

\author{
Jason P. Lynch'1, Stuart B. Mazzone ${ }^{1}$, Matthew J. Rogers ${ }^{1}$, Jaisy J. Arikkatt ${ }^{1}$, \\ Zhixuan Loh', Antonia L. Pritchard ${ }^{2}$, John W. Upham ${ }^{3,4}$ and Simon Phipps ${ }^{1,5}$
}

\author{
Affiliations: \\ ${ }^{1}$ Laboratory of Respiratory Neuroscience and Mucosal Immunity, School of Biomedical Sciences, The \\ University of Queensland, Brisbane, \\ ${ }^{2}$ Oncogenomics, Queensland Institute of Medical Research, Brisbane, \\ ${ }^{3}$ Lung and Allergy Research Centre, School of Medicine, The University of Queensland, Princess Alexandra \\ Hospital, Brisbane, \\ ${ }^{4}$ Dept of Respiratory Medicine, Princess Alexandra Hospital, Brisbane, and \\ ${ }^{5}$ Australian Infectious Diseases Research Centre, The University of Queensland, Brisbane, Australia.
}

Correspondence: S. Phipps, School of Biomedical Sciences, The University of Queensland, St Lucia, Queensland 4072, Australia. E-mail: s.phippsQuqq.edu.au

ABSTRACT The onset, progression and exacerbations of asthma are frequently associated with viral infections of the lower respiratory tract. An emerging paradigm suggests that this relationship may be underpinned by a defect in the host's antiviral response, typified by the impaired production of type I and type III interferons (IFNs). The failure to control viral burden probably causes damage to the lung architecture and contributes to an aberrant immune response, which together compromise lung function.

Although a relatively rare cell type, the plasmacytoid dendritic cell dedicates much of its transcriptome to the synthesis of IFNs and is pre-armed with virus-sensing pattern recognition receptors. Thus, plasmacytoid dendritic cells are specialised to ensure early viral detection and the rapid induction of the antiviral state to block viral replication and spread. In addition, plasmacytoid dendritic cells can limit immunopathology, and promote peripheral tolerance to prevent allergic sensitisation to harmless antigens, possibly through the induction of regulatory T-cells. Thus, this enigmatic cell may lie at an important intersection, orchestrating the immediate phase of antiviral immunity to effect viral clearance while regulating tolerance.

Here, we review the evidence to support the hypothesis that a primary defect in plasmacytoid dendritic function may underlie the development of asthma.

@ERSpublications

A review of the evidence on the role of plasmacytoid dendritic function in the development of asthma http://ow.ly/qieyN

Received: Dec 182012 | Accepted after revision: Feb 042013 | First published online: Feb 212013

Conflict of interest: None declared.

Copyright @ERS 2014 


\section{Introduction}

Asthma is characterised by airway hyperreactivity (AHR) to nonspecific spasmogens, structural alterations to the airway wall and chronic inflammation. The inflammatory response is typically associated with the expression of the T-helper (Th)2-type cytokines interleukin (IL)-4, IL-5, IL-9 and IL-13 [1], which can induce all of the cardinal pathologic features of disease [2,3]. Indeed, the molecular and cellular aspects of the asthmatic reaction, particularly in response to classical allergen provocation, are now fairly well defined, although the emergence of the type-2 innate lymphoid cell (or "nuocytes") has reinforced the concept that innate cells also provide a rich source of Th2-type cytokines [4], in addition to classical Th2 lymphocytes. Despite such progress, our understanding of the processes that promote a Th2-inducing microenvironment and break tolerance to innocuous antigens remains rudimentary and, as a consequence, there is a lack of new immunomodulatory therapies [5].

It is now appreciated that the great majority of exacerbations of asthma are associated with both respiratory virus infections and evidence of Th2 immunity [6-10]. Moreover, epidemiological studies have implicated frequent/severe wheezy lower respiratory tract (LRT) infections as a major risk factor for the onset and progression of asthma in early life [11-15]. Largely determined by a lack of type I interferons (IFN- $\alpha / \beta)$ production in response to virus infection, a new paradigm has emerged in the field linking defective innate antiviral responses in both haematopoietic and nonhaematopoietic cells to increased fragility and damage of the airway epithelium. This defect may also contribute to the development of Th2 immunity, although this concept requires further support. Here, we focus on the plasmacytoid dendritic cell (pDC) and present evidence supporting the hypothesis that a primary defect in the host's "natural type I IFN-producing cell" may underlie the development of asthma. With the capacity to rapidly secrete large amounts of IFN-I and present antigen to naive and memory $\mathrm{CD} 4^{+}$and $\mathrm{CD} 8^{+}$cytotoxic T-lymphocytes, pDCs are at the interface of innate and adaptive antiviral immunity. Curiously, pDCs have also been implicated in mediating tolerance to prevent the induction of allergic asthma $[16,17]$. Thus, this rare and enigmatic cell may lie at an important intersection; orchestrating the immediate phase of antiviral immunity to effect viral clearance while regulating tolerance to self and nonself antigens.

\section{pDCs and their known pattern recognition receptor systems}

pDCs are a relatively rare type of DC that reside predominantly in lymphoid tissues. Both human and murine pDCs express the surface antigen CD45RA and lack the myeloid marker CD11b, although subtle differences exist as human, but not murine, pDCs express the surface markers blood DC antigen (BDCA)-2 (CD303), BDCA-4 (CD304), immunoglobulin-like transcript 7 (ILT7), and the IL-3 receptor- $\alpha$ chain (CD123). In contrast, murine, but not human, pDCs express Siglec H, B220 (CD45R), bone marrow stromal cell antigen 2 (BST2/CD317) and CD11c. As with other antigen-presenting cells, pDCs can acquire and present antigens to T-lymphocytes, although they must first be licensed to do so, e.g. via pattern recognition receptor (PRR) activation $[18,19]$. pDCs also provide help to T-lymphocytes through the provision of co-stimulatory molecules and soluble factors [20]. Indeed, both human and murine pDCs produce prolific amounts of IFN-I (including IFN- $\alpha$, IFN- $\beta$, IFN- $\kappa$ and IFN- $\omega$ ), dedicating $60 \%$ of their transcriptome to IFN production [21] and can release 100-fold more IFN than any other known cell type (3-10 pg per cell of IFN- $\alpha$ ) [22-25]. pDCs also produce type-III IFNs [26] to induce a similar transcriptome in the target cell as IFN-I. Strikingly, pDCs possess the necessary PRRs and signalling intermediaries (e.g. interferon regulatory factor (IRF)7) to recognise viral-derived motifs, and are thus uniquely placed to rapidly sense and respond to viral infections [27-29], even in the absence of cellular infection or viral replication $[29,30]$. This first wave of IFN stimulatory genes establishes the antiviral state, blocks viral replication and facilitates the targeted lysis of infected cells. Among the PRR families, pDCs are most widely acknowledged to express toll-like receptor (TLR)7 and TLR9, which recognise single-stranded (ss)RNA and unmethylated CpG-DNA, respectively. Both of these receptors signal via the adaptor protein MyD88, which, through a signalling cascade, activates transcription of IFN-I, pro-inflammatory cytokines and co-stimulatory molecules. Murine pDCs also express TLR8, which can recognise DNA as well as RNA [31], although it is less clear whether human pDCs express and respond to TLR8 ligands [32, 33]. Activation of the cytosolic RNA sensor retinoic acid-like receptor (RIG)-I, melanoma differentiation-associated protein (MDA)-5 or nucleotide-binding oligomerisation domain-containing protein (NOD)-2, all of which recognise ssRNA (as well as other microbial motifs), can induce IFN-I production by pDCs [32, 34, 35]. RIG-I has been shown to be functional in pDCs but only in the absence of TLR responsiveness [35]; however, RIG-I deficiency does not affect IFN-I or IL-6 production in response to infection with ssRNA viruses, suggesting a compensatory mechanism [36]. Of note, pDCs also express the receptor for advanced glycation end-products (RAGE), which has been associated in two genome-wide association studies as a risk factor for poor lung function $[37,38]$. In an elegant study, the RAGE ligand (high mobility group box 1 protein (HMGB1)) was shown to facilitate viral nucleic acid recognition and optimal IFN-I production 
following activation of TLRs and RIG-I-like receptors (RIG-I and MDA-5) alike [39, 40]. TLR9-induced responses are diminished in RAGE- or HMGB1-null pDCs, which may relate to altered trafficking and a lack of retention of the PRR ligand in the endosome [40]. Whether the RAGE/HMGB1 axis contributes to the activation of TLR7 remains unresolved. Exogenous HMGB1 can inhibit TLR9-mediated IFN-I secretion by pDCs [41], although others have reported that HMGB1 blockade decreases CpG-induced IFN-I production [42]. These conflicting reports may relate to post-translational modifications of HMGB1 which can change its functional activity [43]. In addition to TLR9, pDCs can also detect microbial DNA via the cytosolic helicase DHX36 (DExD/H-box helicase 36) which, like TLR9, employs the MyD88-IRF7 signal transduction cascade to induce IFN-I production [44]. Less is known about the expression of other PRRs, such as the NOD-like receptor family, although the ability to secrete mature IL-1 $\beta$ and IL-18 suggests that pDCs are capable of forming an active inflammasome [45-47]; further studies are warranted to investigate the nature of the inflammasome(s) in pDCs.

\section{Alterations in peripheral pDCs in allergic disorders and effect of allergen challenge}

The earliest studies that sought to determine whether pDC numbers are altered in atopic or asthmatic individuals were performed after observations in the late 1990s, and suggested that the DC1 subtype of DCs promoted Th1 responses and the DC2 subtype (with phenotypic characteristics of pDCs, i.e. human leukocyte antigen (HLA)-DR ${ }^{+}$CD11 $\mathrm{c}^{-} \mathrm{CD} 123^{+}$) promoted Th2 responses, especially when cultured with IL-3 [48, 49]. Congruent with this, UCHIDA et al. [50] reported that the number of HLA-DR ${ }^{+}$CD11c CD123 "DC2" cells was approximately twice as high in the peripheral blood of atopic as compared to healthy subjects. These findings were later confirmed in both atopic and nonatopic asthmatics compared to healthy controls [51, 52]. With the advent of reagents to detect BDCA antigens, the use of CD123 ${ }^{\text {high }}$ CD $11 c^{-}$to identify pDCs declined, and DC subsets were increasingly redefined as DC1 (BDCA-1/CD1c ${ }^{+}$), DC2 (BDCA-3/CD $\left.141^{+}\right)$and pDC (BDCA-2/CD303). Nevertheless, this new phenotyping strategy once again confirmed that circulating pDCs are significantly greater in asthmatics as compared to healthy controls [52].

In response to allergen challenge, pDC numbers increase moderately in the lung and decrease in the periphery. When the bronchial mucosa is sampled $6 \mathrm{~h}$ post-allergen challenge, myeloid DCs $\left(\mathrm{CD} 1 \mathrm{c}^{+} \mathrm{HLA}^{-}\right.$ $\mathrm{DR}^{+}$) are increased, but $\mathrm{pDC}$ numbers are unchanged [53]; however, analysis of bronchiolar lavage fluid and sputum at $24 \mathrm{~h}$ post-segmental allergen challenge found an increase in pDCs [54, 55]. Even after accounting for the different methodologies employed across these studies, the overall picture from asthmatic adults suggests that pDCs are elevated in the periphery and are recruited into the airways in response to allergen challenge. At present, the mechanistic basis of this observation is unclear; however, one possibility is that the inability of asthmatics to produce sufficient IFN-I in the airways in response to infection may fail to activate the negative regulatory feedback loop that exists to homeostatically regulate pDC numbers [56].

In contrast to adults, children with allergic asthma tend to have lower numbers of pDCs in the periphery [57]. Analysis of frozen peripheral blood mononuclear cells collected from children aged 6 or 7 years who had had a wheezing episode associated with a severe respiratory syncytial virus (RSV) infection necessitating hospitalisation in the first year of life found that levels of BDCA $-2^{+}$pDCs were $\sim 50 \%$ lower in the children who were subsequently diagnosed with asthma [58]. In light of the association between wheezy LRT infections in early life and later diagnosis of asthma, UPHAM et al. [59] prospectively examined pDC numbers in peripheral blood in infancy. Intriguingly, pDC numbers were inversely associated with LRT infections and physician-diagnosed asthma at age 5 years [59]. Thus, higher pDC numbers in infancy appear to be protective against asthma inception. Whether this observation relates to a defect in the development and maturation of pDCs or is reflective of a greater infiltration into the airways in response to respiratory allergen challenge or virus infection to lower circulating pDCs [54, 60-62] remains to be determined. However, it is important to note that blood sampling was deferred for 2 weeks in those children who were unwell, suggesting that this did not account for the lower pDC count.

Confirmation of these findings, together with a greater understanding of the molecular basis of this defect, is now of paramount importance. pDC numbers and rhinovirus-stimulated IFN-I responses of peripheral blood mononuclear cells (PBMCs) are greater at 1 month of age and again by 6 months of age as compared with data obtained "at birth" [63,64], raising the possibility that an ontogenic defect may exist in "at risk of asthma" infants. Do low pDC numbers arise from a maturation defect? Alternatively, perhaps there is greater differentiation of pDCs to conventional DCs $[65,66]$ ? It will also be important to determine the influence of environmental factors, such as maternal smoking, gut bacterial colonisation and diet, on the ontogeny of pDCs in early life. Although not in a neonatal setting, it was recently shown that antibiotic treatment of mice (which typically increases the magnitude of allergic inflammation in mouse models of asthma) increases the susceptibility to influenza virus infection-associated damage in the airways [67], an effect linked to lower IRF7 expression in lung macrophages. Should alterations to the microbiota affect IRF7 
expression in pDCs, then this would profoundly affect the induction of antiviral immunity, given the importance of pDCs in the initiation of the immediate IFN-I response (see later).

pDCs from allergic or asthmatic subjects generate aberrant IFN-I responses

To evaluate whether the innate antiviral response is impaired in allergic or asthmatic subjects, multiple laboratories have measured the release of IFN-I and -III, or biomarkers thereof, from peripheral blood leukocytes in response to various TLR stimuli or virus infection. Newcastle disease virus-induced IFN-I release is impaired in PBMCs (which include pDCs) of allergic asthmatic as compared to nonallergic asthmatic children [68] and adults [69]. Intriguingly TLR7- but not TLR3-induced transcription of antiviral molecules and release of the chemokine IFN- $\gamma$-induced protein-10 from PBMCs is reduced in atopic adolescents with mild-to-moderate asthma, as compared with healthy controls [70]. Although it was not directly established that the defect was intrinsic to pDCs, others have shown that the capacity of allergic or asthmatic donor pDCs to produce IFN-I (and, where examined, IFN-III) is impaired in response to TLR9/CpG or virus (influenza or rhinovirus) stimulation [71-75]. Defects in pDC responsiveness may also arise from genetic abnormalities such as single nucleotide polymorphisms. Recent studies have demonstrated that TLR7 and TLR8 single nucleotide polymorphisms have a strong association with asthma across diverse populations $[76,77]$, although how these impact upon expression and protein function is unknown at present. Collectively, these findings suggest that the defect in asthma may relate to the TLR7 and TLR9 pathway (which both signal via MyD88-IRF7) and not the TLR3 pathway (which preferentially signals via IRF3-TIR (Toll-IL-1-resistant)domain-containing adapter-inducing IFN- $\beta$ ).

The high-affinity Fc receptor for IgE is a negative regulator of pDC-derived IFN-I production An impressive body of work now suggests that: 1) expression of the high-affinity IgE receptor, FceRI $\alpha$, on pDCs is greater in allergic and/or asthmatic subjects [75]; 2) FceRI $\alpha$ expression on pDCs is inversely proportional to IFN-I/-III production [72, 73, 75]; and 3) cross-linking of FceRI $\alpha$ impedes the capacity of pDCs to release IFN-I and IFN-III [74, 75]. Segmental allergen challenge in human subjects reduces the production of IFN-I in BDCA- $4^{+}$pDCs purified from PBMCs, supporting the notion that IgE-mediated signalling pathways operate in vivo to modulate pDC function [78]. Mechanistically, anti-IgE has been shown to downregulate TLR9 expression by inducing tumour necrosis factor production from pDCs [79]. Moreover, cross-linking of FceRI on pDCs can activate ILT7, an inhibitory receptor bearing an immunoreceptor tyrosine-based activation motif, to negatively regulate IFN-I production by pDCs (fig. 1) [80]. The IFN-stimulated antigen BST2 has since been identified as a ligand of ILT7, suggesting a negative feedback loop to prevent excessive IFN-I production [81]. Indeed, BST2 ligation of ILT7 suppresses influenza virus (TLR7)- or CpG (TLR9)-triggered release of IFN-I by pDCs [81]. Similarly, activation of either human BDCA-2 or murine Siglec H induces an inhibitory signal through spleen tyrosine kinase (Syk) to attenuate IFN-I production [81]. A recent report found that hepatitis $\mathrm{C}$ viral glycoprotein e2 ligates BDCA-2 to inhibit pDC production of IFN-I and III [82], while elevated phosphorylation of Syk is associated with attenuated IFN-I production by HIV-stimulated pDCs [83]. It will be important to determine whether respiratory viruses, and in particular those associated with the onset of asthma, are able to engage BDCA-2 or ILT7 to evoke inhibitory signals that suppress IFN-I release. The pharmaceutical industry has long sought to develop Syk kinase inhibitors to prevent IgE-mediated mast cell degranulation, and in a stroke of serendipity, it now seems evident that this strategy may also remove the negative tonic on pDCs, thus promoting the release of IFNs to induce antiviral immunity and, feasibly, dampening aberrant Th2 responses.

In light of these experimental data, therapies aimed at decreasing FceRI expression might also enhance antiviral immunity. Encouragingly, immunoneutralisation of circulating $\operatorname{IgE}$ with anti-IgE therapy (omalizumab) has been shown to decrease the expression of FceRI on human pDCs in severe asthma [84], although an important and unresolved question is whether this would increase IFN-I production in response to virus stimulation. It is also worth noting that subcutaneous allergen immunotherapy has been found to heighten IFN-I production by CpG-stimulated pDCs [85], although this was not associated with a fall in pDC FceRI expression or serum IgE. Blockade of Th2 responses may also be beneficial since both IL-4 and IL-13 promote B-lymphocyte class switching to IgE. Moreover, IL-4 promotes apoptosis of human pDCs, downregulates major histocompatibility complex class I expression [86], and both IL-4 and IL-13 can diminish CpG-induced IFN-I production. The molecular mechanism(s) by which activation of the IL-4 receptor- $\alpha$ attenuates TLR signalling remains to be determined. Collectively, these data suggest that Th2 immune responses dampen the effectiveness of pDCs to produce antiviral cytokines, and may help to explain the sizeable increase in asthma risk in those subjects who are both sensitised in early life and experience severe or frequent LRT infections [87]. 


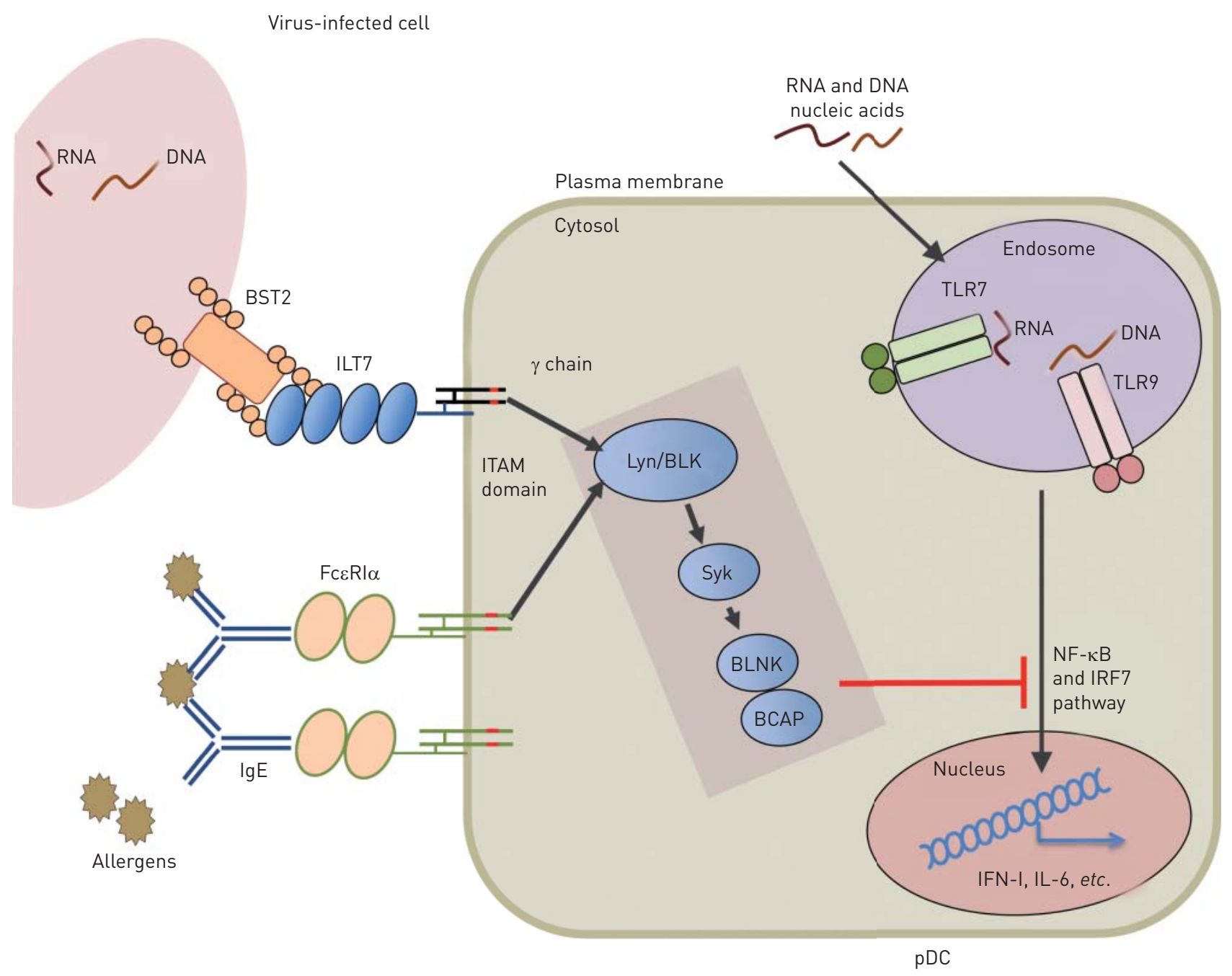

FIGURE 1 Negative regulation of plasmacytoid dendritic cell (pDC) antiviral function by inhaled allergens and virus-infected cells. Allergen-induced crosslinking of FceRI $\alpha$ and/or engagement of immunoglobulin-like transcript (ILT)7 by bone marrow stromal cell antigen (BST) 2 on pDCs activates the B-cell receptor (BCR)-like pathway via FcERI $\gamma$, which contains a transmembrane immunoreceptor tyrosine-based activating motif (ITAM) domain. FcERI $\gamma$-ILT7 or FceRI $\gamma$-FceRI $\alpha$ complexes drive the BCR-like signal transduction cascade which involves Lyn kinase, B-lymphoid tyrosine kinase (BLK) and spleen tyrosine kinase (Syk), and the B-lymphocyte-specific adaptor protein B-cell linker (BLNK) and B-cell adaptor protein (BCAP). Activation of the BCR-like pathway inhibits type-I interferon (IFN-I) and cytokine production in response to DNA or RNA virus activation of the Toll-like receptor (TLR)7/9-MyD88 signalling cascade. NF- $\mathrm{KB}$ : nuclear factor $\kappa \mathrm{B}$; IRF: interferon regulatory factor; IL: interleukin.

Although pDC numbers appear to be greater in asthmatics in later life and lower in high-risk infants in early life, more studies are required to substantiate these important findings. The available data support the notion that IFN-I production by pDCs is impaired in subjects with atopic dermatitis, allergic rhinitis, and allergic and nonallergic asthma, irrespective of age. Despite recent evidence of heightened production of T-lymphocyte derived Th2 cytokines to allergen- or rhinovirus-stimulated PBMCs in the absence of pDCs (or presence of an IFN- $\alpha / \beta$ receptor antagonist) $[88,89]$, it remains an open question whether defective pDCs directly contribute to the development of Th2 responses in vivo or merely fail to constrain them.

\section{Regulation of TLR7-mediated responses}

Hyper- and hypo-IFN responses may underlie a number of pathologies, including microbial infections, tumour development, autoimmune diseases and chronic inflammatory diseases, including asthma, and, as a consequence, rapid advances have occurred with regard to our understanding of the molecular processes that regulate endosomal TLRs [90]. For example, it is now appreciated that TLR7 and TLR9 translocate from the endoplasmic reticulum to a specialised lysosome-like organelle prior to IFN-I synthesis. This process is in part orchestrated by the endoplasmic reticulum-associated molecule UNC93b [91], and involves a number of lysosome-related organelle trafficking and biogenesis proteins including adapterrelated protein complex-3, Hermansky-Pudlak syndrome proteins biogenesis of lysosome-related organelles 
complex (BLOC)-1 and BLOC-2 and the solute channel protein Slc15a4 [92, 93]. While evidence is emerging in systemic lupus erythematosus to suggest that $\operatorname{defect}(\mathrm{s})$ in the lysosomal machinery contributes to dysregulated IFN-I responses [94, 95], no data are yet available with regard to asthma, which is perhaps surprising in light of the substantial evidence showing that both TLR7 and TLR9 responses of pDCs are blunted.

In an elegant study, activation of RIG-I-like receptor-activated IRF3 was found to interfere with TLRinduced transcription factor complexes, impairing gene expression of IL-12b (which encodes the p40 subunit) [96]. The authors suggest that this has important consequences with respect to polymicrobial infections, although it may operate during single pathogen exposures that can independently activate multiple PRRs. Of note, IRF3 is employed by several PRRs including MDA-5, NOD2, RIG-I and TLR3, all expressed by pDCs and activated by viral RNA. We have recently observed that infection of IRF3-deficient mice with pneumonia virus of mice (PVM) led to a hyper-IFN-I response (S. Phipps, unpublished observations), while NOD2 activation by the bacterial ligand muramyl dipeptide can suppress CpG stimulated IFN-I secretion by liver pDCs [34], suggesting that IRF3 might also impair TLR7/TLR9/IRF7-mediated responses by pDCs. It remains to be formally determined that cross-interference occurs in pDCs, but it is tantalising to speculate that microbiota of the gut or the lung, or an existing pathogenic infection, may affect the functional responses of pDCs to a respiratory viral infection. Finally, control of IRF7 gene expression via the translational repressors $4 \mathrm{E}-\mathrm{BP} 1$ and $4 \mathrm{E}-\mathrm{BP} 2$ can dramatically alter the magnitude of IFN-I production and, consequently, viral clearance [97]. It will be fascinating to learn whether modulation of these influential repressors contributes to the IFN-I defect in asthma.

\section{Viral subversion of TLR7 responses}

TLR7 activation and induction of IFN-I expression can occur in the absence of viral replication [98, 99]. In addition to the classical delivery of material via the endocytic and micropinocytic pathways to endosomes, it is now recognised that cytosolic viral RNA intermediates can be delivered to endosome-sequestered TLRs via the process of autophagy [100], which can be accelerated via HMGB1 ligation of RAGE [43, 101]. Thus, diminished cellular function that typically occurs following ultraviolet (UV) inactivation of virus may not simply reflect the requirement for virus replication per se; rather, inactivation may affect the delivery of the cargo and, hence, the PRRs by which it is first recognised [102]. This may in part explain why some investigators have reported UV-inactivation of RSV to diminish IFN-I production by pDCs [103, 104]. The ability of clinical isolates of RSV (and metapneumovirus (MPV)) to attenuate TLR7/TLR9-induced IFN-I responses in pDCs has been consistently shown in human and murine models [104-106]. The infection of pDCs by RSV was implied when cell surface expression of the viral F protein was observed, which was later confirmed using green fluorescent protein-labelled RSV; although the fraction of infected pDCs was extremely low $[103,107]$.

Somewhat surprisingly, few studies have investigated pDC-rhinovirus interactions, irrespective of the context of asthma. Pritchard et al. [88] demonstrated that rhinovirus-16-induced IFN-I release is dramatically reduced when pDCs are depleted from healthy PBMC cultures, inferring that pDCs recognise rhinovirus. As in studies with RSV, TLR7-induced IFN-I production from PBMC or cord blood pDCs is reduced by $\sim 50 \%$ when cultured with rhinovirus strain $1 \mathrm{~b}$ prior to stimulation [63]. In light of the important role of respiratory viruses in the onset, progression and exacerbations of asthma, a priority for the future will be to unravel the mechanisms by which $\mathrm{pDC}$ responses can be subverted by RSV, rhinovirus, MPV (and other viruses), and to determine whether this process is enhanced in allergen-sensitised or other at risk of asthma infants.

\section{Evidence from mouse models that pDCs contribute to host defence against respiratory virus infection}

Recently generated pDC "knock-in" mice harbouring the diphtheria toxin receptor have enabled the inducible depletion of pDCs via the administration of diphtheria toxin [108, 109]. Using these novel transgenic mice, it was definitively shown that pDCs provide the immediate source of IFN-I in the very early phase of infection to slow viral propagation and contribute to cytotoxic T-lymphocyte responses [108, 109]. At present, these strains have not been employed in conjunction with a respiratory viral infection. However, two studies have investigated the effect of antibody-mediated pDC depletion on the course of RSV infection, and both reported an attenuated early IFN-I response and increased viral burden in the absence of pDCs $[110,111]$. Of note, Th2 responses and the magnitude of immunopathology to primary human RSV infection are enhanced following infection of pDC-depleted or TLR7-deficient mice [110, 112]. These early studies may require verification, since it is now apparent that the use of less than three antigens (e.g. reliance on CD11c and B220 alone) is insufficient to discriminate pDCs using flow cytometry. Additionally, BST2 (also known as PDCA-1) is upregulated on various cell types in response to infection [113], potentially confounding some analyses or studies where the depleting antibody has been used for protracted periods of time. 


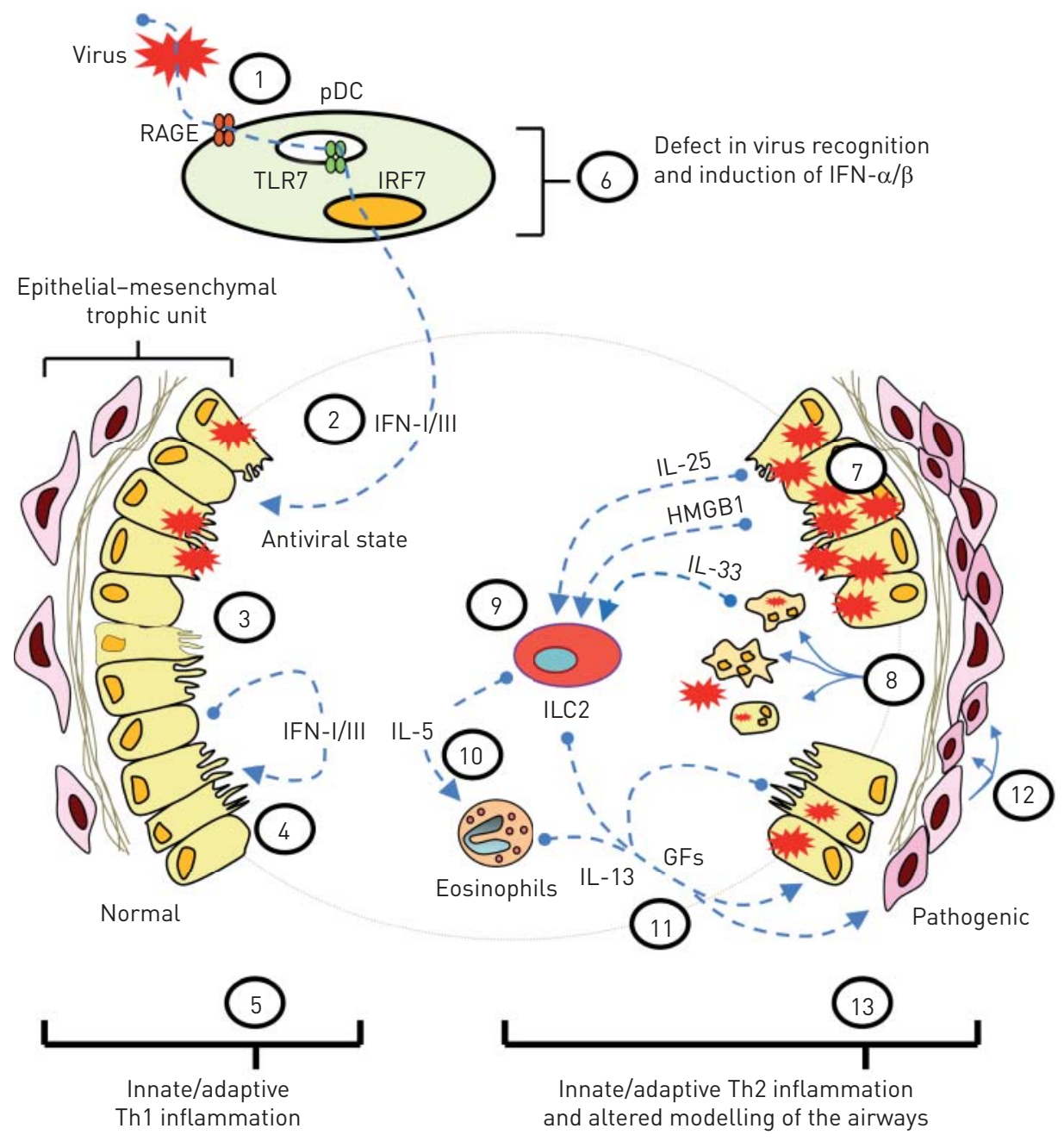

FIGURE 2 Impaired type I and type III interferon (IFN) production consequent to a genetic/functional defect(s) in virussensing pattern recognition receptors (PRRs) expressed by plasmacytoid dendritic cells (pDCs) promotes a type-2 immune response and airway remodelling. In healthy individuals, pDCs recognise an invading respiratory virus (e.g. respiratory syncytial virus or rhinovirus) through a receptor for advanced glycation end-products (RAGE)-Toll-like receptor (TLR)7-interferon regulatory factor (IRF)7 axis (step 1), and rapidly produce vast amounts of type I and III IFNs (step 2). IFN secretion by pDCs acts in a paracrine manner to establish the immediate phase of the antiviral state in nonspecialised antiviral cells (step 3). The airway epithelium fortifies itself against the virus by various means, including the production of IFNs, which act locally to support neighbouring cells (step 4). An appropriate T-helper (Th) 1 response is generated, the virus is cleared and tissue homeostasis is restored (step 5). In contrast, a genetic/functional defect(s) in virus-sensing PRRs expressed by pDCs (step 6) fails to induce the antiviral state in airway epithelial cells, increasing viral burden (step 7) and injuring the airway epithelium, which becomes necrotic leading to the formation of creola bodies in the lumen (step 8). The damaged epithelium releases alarmins and pro-Th2 instructive cytokines to promote the recruitment and expansion of type-2 innate lymphoid cells (step 9), which support eosinophil survival through the production of interleukin (IL)-5 (step 10). These Th2-type effector cells, together with the injured epithelium, promote wound repair through the secretion of various growth factors (GFs) (step 11). In susceptible individuals, this cycle is repeated upon subsequent infections, leading to airway remodelling (step 12) and chronic Th2-type inflammation (step 13). HMGB1: high-mobility group box 1 protein; ILC2: type-2 innate lymphoid cell (or nuocyte).

We have elected to use the rodent-specific pneumovirus (PVM), which propagates in mice, allowing low inoculums of 5 plaque-forming units (PFU) to be used (in contrast to human RSV, where typically between $10^{5}$ and $10^{7} \mathrm{PFU}$ are administered). Importantly, the use of a physiologically relevant low dose of inoculum is more likely to ensure that the pertinent PRRs and cell types are activated in a spatiotemporal manner akin to a natural infection. This concept is supported by the observation that pDC-mediated control of viral load only occurs at a low dose of virus [108]. We have demonstrated that the immediate response to low-dose PVM is TLR7 dependent, and that TLR7 on pDCs mediates the early IFN response to control viral spread (fig. 2) [114]. In the absence of an early antiviral response, TLR7 deficiency leads to airway epithelial cell sloughing and denudation of the basement membrane. Moreover, this was associated with increased expression of the tissue alarmin and 
Th2-instructive cytokine IL-33, the infiltration of type 2 innate lymphoid cells and elevated IL-13 production. Virus challenge at 7 weeks of age induced all the hallmark features of asthma, including AHR and increases in airway smooth muscle mass. Furthermore, sensitisation with the cockroach antigen during primary infection of TLR7-deficient mice markedly increased the magnitude of allergic airways inflammation (Phipps et al., unpublished observations). Our data suggest that TLR7, pneumovirus infection and a clinically relevant allergen interact to establish an aberrant adaptive response that underlies virus-induced asthma exacerbations in later life.

\section{pDC-derived paracrine support for structural cells}

A central question that remains to be addressed is how the absence of pDC-derived paracrine signals (in particular IFN-I and -III) impacts the airway epithelium, the underlying mesenchyme and other cells resident within the airway wall. Pathological analyses of the airway wall reveal the airway epithelium of asthmatics to be highly disorganised, with evidence of sloughing and denudation of the basement membrane, mucus cell metaplasia, airway epithelial cell (AEC) hyperproliferation and an impaired ability to undergo re-epithelisation [5]. This phenotype may, in part, be mediated by a combination of genetic and environmental factors affecting epithelial barrier function [115], immunopathology caused by an aberrant host response or an inability to clear microbial infections. Although some investigators have shown that the airway epithelium itself is unable to produce a robust IFN-I response [116, 117], this phenotype has not been reproduced by others $[118,119]$. We speculate that it is the absence of a robust pDC response that primarily underlies the antiviral defect in asthma. The unique ability of pDCs to rapidly secrete large amounts of IFN-I suggests that they orchestrate the early-phase response, whereby the antiviral state is primed in nonspecialised cells such as AECs (fig. 2). Thus, in the absence of paracrine signals produced by pDCs, the AEC will be ill-equipped to: 1) recognise the invading pathogen; 2) recruit and signal its deletion by cytotoxic immune cells; and 3) produce IFN-I to amplify the antiviral response in neighbouring cells.

In addition, the attenuated IFN-I response may impede the repair process. Using a skin model of reepithelisation, pDC-derived IFN-I was recently found to be critical for the effective repair of the epidermis [120]. Thus, it is highly conceivable that defects in the pDC pool will affect not only susceptibility to infection but also the ability of the epithelial-mesenchymal trophic unit to repair itself. Additionally, changes to the epithelium might promote the development of Th2 immunity by modulating the underlying network of DC cells $[121,122]$. For example, house dust mite-induced epithelial-derived IL- $1 \alpha$ can activate an autocrine loop to induce the Th2-instructive cytokines IL-33 and granulocyte-macrophage colonystimulating factor, which license local DCs to promote Th2 lung inflammation [123]. Thus, damage to the epithelium caused by the proteolytic activity of allergens, excessive immunopathology and/or respiratory viral infection-associated cytopathology induces the release of tissue alarmins IL- $1 \alpha$, HMGB1 and IL-33, which appear to promote the development of Th2 immunity (fig. 2). Teleologically, this response may have developed to initiate tissue repair, but following repeated environmental insult may lead to tissue remodelling [124-126]. We postulate that a defect in the pDC compartment may establish a pro-Th2 microenvironment as a consequence of increased tissue damage and release of alarmins by structural cells. In this paradigm, one can envisage how a viral infection and encounter with an allergen in a susceptible host (e.g. with a defect in the pDC compartment) may collude to establish allergic-specific Th2-type immunity.

pDCs and "noninfectious" asthma

Mouse models have decisively demonstrated the requirement for conventional DCs to elicit both allergic sensitisation and the effector/challenge phase [127]. However, in a seminal study, DE HEER et al. [16] demonstrated that pDCs contribute to peripheral tolerance. Whereas inhalation of the model allergen ovalbumin (OVA) in the absence of an adjuvant leads to tolerance, prior depletion of pDCs leads to the development of allergic sensitisation and pathologic features of asthma. Conversely, the adoptive transfer of OVA-pulsed pDCs prior to a fully immunogenic asthma protocol suppresses the magnitude of the allergic response [16], an effect subsequently shown to be independent of IFN-I, and instead mediated via the coinhibitory receptor programmed death-1 and its cognate ligand programmed death ligand 1 [128]. Intriguingly, immunoneutralisation of the cytokine osteopontin during intraperitoneal OVA/alum sensitisation also attenuates allergic sensitisation through a mechanism involving pDCs, apparently though a reduction of the regulatory capacity of pDCs [17]. However, the molecular pathway that underpins this response requires further elucidation. While $\mathrm{pDCs}$ are able to acquire antigen and traffic to the draining lymph nodes they appear less able to prime naive T-lymphocytes to proliferate [16, 17]. This led De HeER et al. [16] to hint that pDCs may mediate their tolerogenic properties through the induction of regulatory $\mathrm{T}$ lymphocytes (Tregs), although this was not directly demonstrated. However, a recent report showed specific subsets of pDCs $\left(\mathrm{CD} 8 \alpha^{+} \beta^{+}\right.$or $\mathrm{CD} 8 \alpha^{+} \beta^{-}$but not $\left.\mathrm{CD} 8 \alpha^{-} \beta^{-}\right)$are able to induce the differentiation of regulatory Tregs to abrogate inflammation and block AHR [129]. The tolerogenic pDC subsets expressed aldehyde dehydrogenase which in part catalyses retinol to retinoic acid, which, together with transcription growth 
factor- $\beta$, supports the development of FoxP $3^{+} \mathrm{CD} 4^{+}$Tregs. It is noteworthy that the adoptive transfer of the nontolerogenic pDC subset (pulsed with OVA) is able to initiate allergic airways inflammation and AHR. It will be important to determine whether human equivalents to these subsets exist and whether they exhibit similar functions. Indeed, other pDC subsets have been proposed based on the expression of CCR9 [130], CX3CR1 [131], CD9 [132] or the balance of IFN-I:IFN-III production [133]. Further work is now needed to confirm their distinct functional repertoires and to relate these to normal and pathogenic process.

\section{Conclusions}

In summary, evidence from clinical and murine studies suggests that TLR7 deficiency or defects in the number of circulating pDCs is associated with the development of Th2-associated lung inflammation in response to virus infection. In adults, $\mathrm{pDCs}$ are twice as prevalent in the circulation of individuals with atopic dermatitis, allergic rhinitis or asthma than healthy controls. Moreover, these pDCs are refractory to TLR ligand or virus stimulation, a phenotype that may relate to the activation of FceR1and other surface receptors that activate Syk kinase. Targeting the pathways that establish a state of pDC hyporesponsiveness should now be a priority for the treatment of asthma, since restoration of $\mathrm{pDC}$ function may help redress the defective antiviral immune response, reduce immunopathology and increase tolerance to harmless antigens.

\section{References}

Kay AB. Allergy and allergic diseases. Second of two parts. N Engl J Med 2001; 344: 109-113.

Wills-Karp M, Luyimbazi J, Xu X, et al. Interleukin-13: central mediator of allergic asthma. Science 1998; 282: 2258-2261.

3 Zhu Z, Homer RJ, Wang Z, et al. Pulmonary expression of interleukin-13 causes inflammation, mucus hypersecretion, subepithelial fibrosis, physiologic abnormalities, and eotaxin production. J Clin Invest 1999; 103: 779-788.

4 Barlow JL, McKenzie AN. Nuocytes: expanding the innate cell repertoire in type-2 immunity. J Leukoc Biol 2011; 90: 867-874.

5 Holgate ST. Has the time come to rethink the pathogenesis of asthma? Curr Opin Allergy Clin Immunol 2010; 10: $48-53$.

6 Nicholson KG, Kent J, Ireland DC. Respiratory viruses and exacerbations of asthma in adults. BMJ 1993; 307: 982-986.

7 Johnston SL, Pattemore PK, Sanderson G, et al. The relationship between upper respiratory infections and hospital admissions for asthma: a time-trend analysis. Am J Respir Crit Care Med 1996; 154: 654-660.

8 Johnston SL, Pattemore PK, Sanderson G, et al. Community study of role of viral infections in exacerbations of asthma in 9-11 year old children. BMJ 1995; 310: 1225-1229.

9 Message SD, Laza-Stanca V, Mallia P, et al. Rhinovirus-induced lower respiratory illness is increased in asthma and related to virus load and Th1/2 cytokine and IL-10 production. Proc Natl Acad Sci USA 2008; 105: 13562-13567.

10 Calderon C, Rivera L, Hutchinson P, et al. T-cell cytokine profiles are altered in childhood asthma exacerbation. Respirology 2009; 14: 264-269.

11 Jackson DJ, Gangnon RE, Evans MD, et al. Wheezing rhinovirus illnesses in early life predict asthma development in high-risk children. Am J Respir Crit Care Med 2008; 178: 667-672.

12 Khetsuriani N, Kazerouni NN, Erdman DD, et al. Prevalence of viral respiratory tract infections in children with asthma. J Allergy Clin Immunol 2007; 119: 314-321.

13 Kusel MM, de Klerk NH, Kebadze T, et al. Early-life respiratory viral infections, atopic sensitization, and risk of subsequent development of persistent asthma. J Allergy Clin Immunol 2007; 119: 1105-1110.

14 Sigurs N, Bjarnason R, Sigurbergsson F, et al. Respiratory syncytial virus bronchiolitis in infancy is an important risk factor for asthma and allergy at age 7. Am J Respir Crit Care Med 2000; 161: 1501-1507.

15 Stein RT, Sherrill D, Morgan WJ, et al. Respiratory syncytial virus in early life and risk of wheeze and allergy by age 13 years. Lancet 1999; 354: 541-545.

16 De Heer HJ, Hammad H, Soullié T, et al. Essential role of lung plasmacytoid dendritic cells in preventing asthmatic reactions to harmless inhaled antigen. J Exp Med 2004; 200: 89-98.

17 Xanthou G, Alissafi T, Semitekolou M, et al. Osteopontin has a crucial role in allergic airway disease through regulation of dendritic cell subsets. Nat Med 2007; 13: 570-578.

18 Mouriès J, Moron G, Schlecht G, et al. Plasmacytoid dendritic cells efficiently cross-prime naive T cells in vivo after TLR activation. Blood 2008; 112: 3713-3722.

19 Young LJ, Wilson NS, Schnorrer P, et al. Differential MHC class II synthesis and ubiquitination confers distinct antigen-presenting properties on conventional and plasmacytoid dendritic cells. Nat Immunol 2008; 9: 1244-1252.

20 Cervantes-Barragan L, Lewis KL, Firner S, et al. Plasmacytoid dendritic cells control T-cell response to chronic viral infection. Proc Natl Acad Sci USA 2012; 109: 3012-3017.

21 Ito T, Kanzler H, Duramad O, et al. Specialization, kinetics, and repertoire of type 1 interferon responses by human plasmacytoid predendritic cells. Blood 2006; 107: 2423-2431.

22 Fitzgerald-Bocarsly P, Dai J, Singh S. Plasmacytoid dendritic cells and type I IFN: 50 years of convergent history. Cytokine Growth Factor Rev 2008; 19: 3-19.

23 Liu YJ. IPC: professional type 1 interferon-producing cells and plasmacytoid dendritic cell precursors. Annu Rev Immunol 2005; 23: 275-306.

24 Gilliet M, Cao W, Liu YJ. Plasmacytoid dendritic cells: sensing nucleic acids in viral infection and autoimmune diseases. Nat Rev Immunol 2008; 8: 594-606.

25 Honda K, Yanai H, Negishi H, et al. IRF-7 is the master regulator of type-I interferon-dependent immune responses. Nature 2005; 434: 772-777. 
26 Coccia EM, Severa M, Giacomini E, et al. Viral infection and Toll-like receptor agonists induce a differential expression of type I and $\lambda$ interferons in human plasmacytoid and monocyte-derived dendritic cells. Eur J Immunol 2004; 34: 796-805.

27 Izaguirre A, Barnes BJ, Amrute S, et al. Comparative analysis of IRF and IFN-alpha expression in human plasmacytoid and monocyte-derived dendritic cells. J Leukoc Biol 2003; 74: 1125-1138.

28 Kawai T, Sato S, Ishii KJ, et al. Interferon- $\alpha$ induction through Toll-like receptors involves a direct interaction of IRF7 with MyD88 and TRAF6. Nat Immunol 2004; 5: 1061-1068.

29 Barchet W, Cella M, Odermatt B, et al. Virus-induced interferon $\alpha$ production by a dendritic cell subset in the absence of feedback signaling in vivo. J Exp Med 2002; 195: 507-516.

30 Beignon AS, McKenna K, Skoberne M, et al. Endocytosis of HIV-1 activates plasmacytoid dendritic cells via Tolllike receptor-viral RNA interactions. J Clin Invest 2005; 115: 3265-3275.

31 Martinez J, Huang X, Yang Y. Toll-like receptor 8-mediated activation of murine plasmacytoid dendritic cells by vaccinia viral DNA. Proc Natl Acad Sci USA 2010; 107: 6442-6447.

32 Ablasser A, Poeck H, Anz D, et al. Selection of molecular structure and delivery of RNA oligonucleotides to activate TLR7 versus TLR8 and to induce high amounts of IL-12p70 in primary human monocytes. J Immunol 2009; 182: 6824-6833.

33 Cervantes JL, Weinerman B, Basole C, et al. TLR8: the forgotten relative revindicated. Cell Mol Immunol 2012; 9: 434-438.

34 Castellaneta A, Sumpter TL, Chen L, et al. NOD2 ligation subverts IFN- $\alpha$ production by liver plasmacytoid dendritic cells and inhibits their $\mathrm{T}$ cell allostimulatory activity via B7-H1 up-regulation. J Immunol 2009; 183: 6922-6932.

35 Kumagai Y, Kumar H, Koyama S, et al. Cutting Edge: TLR-dependent viral recognition along with type I IFN positive feedback signaling masks the requirement of viral replication for IFN- $\alpha$ production in plasmacytoid dendritic cells. J Immunol 2009; 182: 3960-3964.

36 Kato H, Sato S, Yoneyama M, et al. Cell type-specific involvement of RIG-I in antiviral response. Immunity 2005; 23: 19-28.

37 Repapi E, Sayers I, Wain LV, et al. Genome-wide association study identifies five loci associated with lung function. Nat Genet 2010; 42: 36-44.

38 Hancock DB, Eijgelsheim M, Wilk JB, et al. Meta-analyses of genome-wide association studies identify multiple loci associated with pulmonary function. Nat Genet 2010; 42: 45-52.

39 Yanai H, Ban T, Wang Z, et al. HMGB proteins function as universal sentinels for nucleic-acid-mediated innate immune responses. Nature 2009; 462: 99-103.

40 Tian J, Avalos AM, Mao SY, et al. Toll-like receptor 9-dependent activation by DNA-containing immune complexes is mediated by HMGB1 and RAGE. Nat Immunol 2007; 8: 487-496.

41 Popovic PJ, DeMarco R, Lotze MT, et al. High mobility group B1 protein suppresses the human plasmacytoid dendritic cell response to TLR9 agonists. J Immunol 2006; 177: 8701-8707.

42 Dumitriu IE, Baruah P, Bianchi ME, et al. Requirement of HMGB1 and RAGE for the maturation of human plasmacytoid dendritic cells. Eur J Immunol 2005; 35: 2184-2190.

43 Malarkey CS, Churchill ME. The high mobility group box: the ultimate utility player of a cell. Trends Biochem Sci 2012; 37: 553-562.

44 Kim T, Pazhoor S, Bao M, et al. Aspartate-glutamate-alanine-histidine box motif (DEAH)/RNA helicase A helicases sense microbial DNA in human plasmacytoid dendritic cells. Proc Natl Acad Sci USA 2010; 107: 15181-15186.

45 Barr DP, Belz GT, Reading PC, et al. A role for plasmacytoid dendritic cells in the rapid IL-18-dependent activation of NK cells following HSV-1 infection. Eur J Immunol 2007; 37: 1334-1342.

46 Lousberg EL, Diener KR, Fraser CK, et al. Antigen-specific T-cell responses to a recombinant fowlpox virus are dependent on MyD88 and IL-18 and independent of Toll-like receptor 7 (TLR7)- and TLR9-mediated innate immune recognition. J Virol 2011; 85: 3385-3396.

47 Rodriguez Rodrigues C, Cabrini M, Remes Lenicov F, et al. Epithelial cells activate plasmacytoid dendritic cells improving their anti-HIV activity. PLoS One 2011; 6: e28709.

48 Rissoan MC, Soumelis V, Kadowaki N, et al. Reciprocal control of T helper cell and dendritic cell differentiation. Science 1999; 283: 1183-1186.

49 Kadowaki N, Antonenko S, Lau JY, et al. Natural interferon $\alpha / \beta$-producing cells link innate and adaptive immunity. J Exp Med 2000; 192: 219-226.

50 Uchida Y, Kurasawa K, Nakajima H, et al. Increase of dendritic cells of type 2 (DC2) by altered response to IL-4 in atopic patients. J Allergy Clin Immunol 2001; 108: 1005-1011.

51 Matsuda H, Suda T, Hashizume H, et al. Alteration of balance between myeloid dendritic cells and plasmacytoid dendritic cells in peripheral blood of patients with asthma. Am J Respir Crit Care Med 2002; 166: 1050-1054.

52 Spears M, McSharry C, Donnelly I, et al. Peripheral blood dendritic cell subtypes are significantly elevated in subjects with asthma. Clin Exp Allergy 2011; 41: 665-672.

53 Jahnsen FL, Moloney ED, Hogan T, et al. Rapid dendritic cell recruitment to the bronchial mucosa of patients with atopic asthma in response to local allergen challenge. Thorax 2001; 56: 823-826.

54 Bratke K, Lommatzsch M, Julius P, et al. Dendritic cell subsets in human bronchoalveolar lavage fluid after segmental allergen challenge. Thorax 2007; 62: 168-175.

55 Dua B, Watson RM, Gauvreau GM, et al. Myeloid and plasmacytoid dendritic cells in induced sputum after allergen inhalation in subjects with asthma. J Allergy Clin Immunol 2010; 126: 133-139.

56 Swiecki M, Wang Y, Vermi W, et al. Type I interferon negatively controls plasmacytoid dendritic cell numbers in vivo. J Exp Med 2011; 208: 2367-2374.

57 Hagendorens MM, Ebo DG, Schuerwegh AJ, et al. Differences in circulating dendritic cell subtypes in cord blood and peripheral blood of healthy and allergic children. Clin Exp Allergy 2003; 33: 633-639.

58 Silver E, Yin-DeClue H, Schechtman KB, et al. Lower levels of plasmacytoid dendritic cells in peripheral blood are associated with a diagnosis of asthma $6 \mathrm{yr}$ after severe respiratory syncytial virus bronchiolitis. Pediatr Allergy Immunol 2009; 20: 471-476.

59 Upham JW, Zhang G, Rate A, et al. Plasmacytoid dendritic cells during infancy are inversely associated with childhood respiratory tract infections and wheezing. J Allergy Clin Immunol 2009; 124: 707-713. 
60 Farrell E, O'Connor TM, Duong M, et al. Circulating myeloid and plasmacytoid dendritic cells after allergen inhalation in asthmatic subjects. Allergy 2007; 62: 1139-1145.

61 Gill MA, Palucka AK, Barton T, et al. Mobilization of plasmacytoid and myeloid dendritic cells to mucosal sites in children with respiratory syncytial virus and other viral respiratory infections. J Infect Dis 2005; 191: 1105-1115.

62 Gill MA, Long K, Kwon T, et al. Differential recruitment of dendritic cells and monocytes to respiratory mucosal sites in children with influenza virus or respiratory syncytial virus infection. J Infect Dis 2008; 198: 1667-1676.

63 Koumbi LJ, Papadopoulos NG, Anastassiadou V, et al. Dendritic cells in uninfected infants born to hepatitis B virus-positive mothers. Clin Vaccine Immunol 2010; 17: 1079-1085.

64 Kollmann TR, Levy O, Montgomery RR, et al. Innate immune function by Toll-like receptors: distinct responses in newborns and the elderly. Immunity 2012; 37: 771-783.

65 Soumelis V, Liu YJ. From plasmacytoid to dendritic cell: morphological and functional switches during plasmacytoid pre-dendritic cell differentiation. Eur J Immunol 2006; 36: 2286-2292.

66 Liou LY, Blasius AL, Welch MJ, et al. In vivo conversion of BM plasmacytoid DC into CD11b ${ }^{+}$conventional DC during virus infection. Eur J Immunol 2008; 38: 3388-3394.

67 Abt MC, Osborne LC, Monticelli LA, et al. Commensal bacteria calibrate the activation threshold of innate antiviral immunity. Immunity 2012; 37: 158-170.

68 Bufe A, Gehlhar K, Grage-Griebenow E, et al. Atopic phenotype in children is associated with decreased virusinduced interferon- $\alpha$ release. Int Arch Allergy Immunol 2002; 127: 82-88.

69 Gehlhar K, Bilitewski C, Reinitz-Rademacher K, et al. Impaired virus-induced interferon- $\alpha 2$ release in adult asthmatic patients. Clin Exp Allergy 2006; 36: 331-337.

70 Roponen M, Yerkovich ST, Hollams E, et al. Toll-like receptor 7 function is reduced in adolescents with asthma. Eur Respir J 2010; 35: 64-71.

71 Novak N, Allam JP, Hagemann T, et al. Characterization of FceRI-bearing CD123 blood dendritic cell antigen-2 plasmacytoid dendritic cells in atopic dermatitis. J Allergy Clin Immunol 2004; 114: 364-370.

72 Schroeder JT, Bieneman AP, Xiao H, et al. TLR9- and FceRI-mediated responses oppose one another in plasmacytoid dendritic cells by down-regulating receptor expression. J Immunol 2005; 175: 5724-5731.

73 Tversky JR, Le TV, Bieneman AP, et al. Human blood dendritic cells from allergic subjects have impaired capacity to produce interferon- $\alpha$ via Toll-like receptor 9. Clin Exp Allergy 2008; 38: 781-788.

74 Gill MA, Bajwa G, George TA, et al. Counterregulation between the FceRI pathway and antiviral responses in human plasmacytoid dendritic cells. J Immunol 2010; 184: 5999-6006.

75 Durrani SR, Montville DJ, Pratt AS, et al. Innate immune responses to rhinovirus are reduced by the high-affinity IgE receptor in allergic asthmatic children. J Allergy Clin Immunol 2012; 130: 489-495.

76 Møller-Larsen S, Nyegaard M, Haagerup A, et al. Association analysis identifies TLR7 and TLR8 as novel risk genes in asthma and related disorders. Thorax 2008; 63: 1064-1069.

77 Zhang Q, Qian F, Zhou L, et al. Polymorphisms of TLR7 and TLR8 associated with risk of asthma and asthmarelated phenotypes in a southeastern Chinese Han population. J Nanjing Med Univ 2009; 23: 25-32.

78 Schroeder JT, Bieneman AP, Chichester KL, et al. Pulmonary allergic responses augment interleukin- 13 secretion by circulating basophils yet suppress interferon- $\alpha$ from plasmacytoid dendritic cells. Clin Exp Allergy 2010; 40: 745-754.

79 Schroeder JT, Chichester KL, Bieneman AP. Toll-like receptor 9 suppression in plasmacytoid dendritic cells after IgE-dependent activation is mediated by autocrine TNF- $\alpha$. J Allergy Clin Immunol 2008; 121: 486-491.

80 Cao W, Rosen DB, Ito T, et al. Plasmacytoid dendritic cell-specific receptor ILT7-FceRI $\gamma$ inhibits Toll-like receptorinduced interferon production. J Exp Med 2006; 203: 1399-1405.

81 Cao W, Bover L, Cho M, et al. Regulation of TLR7/9 responses in plasmacytoid dendritic cells by BST2 and ILT7 receptor interaction. J Exp Med 2009; 206: 1603-1614.

82 Florentin J, Aouar B, Dental C, et al. HCV glycoprotein E2 is a novel BDCA-2 ligand and acts as an inhibitor of IFN production by plasmacytoid dendritic cells. Blood 2012; 120: 4544-4551.

83 Lo CC, Schwartz JA, Johnson DJ, et al. HIV delays IFN- $\alpha$ production from human plasmacytoid dendritic cells and is associated with SYK phosphorylation. PLoS One 2012; 7: e37052.

84 Chanez P, Contin-Bordes C, Garcia G, et al. Omalizumab-induced decrease of Fc $\xi \mathrm{RI}$ expression in patients with severe allergic asthma. Respir Med 2010; 104: 1608-1617.

85 Tversky JR, Bieneman AP, Chichester KL, et al. Subcutaneous allergen immunotherapy restores human dendritic cell innate immune function. Clin Exp Allergy 2010; 40: 94-102.

86 Tel J, Torensma R, Figdor CG, et al. IL-4 and IL-13 alter plasmacytoid dendritic cell responsiveness to CpG DNA and herpes simplex virus-1. J Invest Dermatol 2011; 131: 900-906.

87 Holt PG, Rowe J, Kusel M, et al. Toward improved prediction of risk for atopy and asthma among preschoolers: a prospective cohort study. J Allergy Clin Immunol 2010; 125: 653-659.

88 Pritchard AL, Carroll ML, Burel JG, et al. Innate IFNs and plasmacytoid dendritic cells constrain Th2 cytokine responses to rhinovirus: a regulatory mechanism with relevance to asthma. J Immunol 2012; 188: 5898-5905.

89 Pritchard AL, White OJ, Burel JG, et al. Innate interferons inhibit allergen and microbial specific $\mathrm{T}(\mathrm{H}) 2$ responses. Immunol Cell Biol 2012; 90: 974-977.

90 Bao M, Liu YJ. Regulation of TLR7/9 signaling in plasmacytoid dendritic cells. Protein Cell 2013; 4: 40-52.

91 Tabeta K, Hoebe K, Janssen EM, et al. The Unc93b1 mutation 3d disrupts exogenous antigen presentation and signaling via Toll-like receptors 3, 7 and 9. Nat Immunol 2006; 7: 156-164.

92 Sasai M, Linehan MM, Iwasaki A. Bifurcation of Toll-like receptor 9 signaling by adaptor protein 3. Science 2010; 329: 1530-1534.

93 Blasius AL, Arnold CN, Georgel P, et al. Slc15a4, AP-3, and Hermansky-Pudlak syndrome proteins are required for Toll-like receptor signaling in plasmacytoid dendritic cells. Proc Natl Acad Sci USA 2010; 107: 19973-19978.

94 Han JW, Zheng HF, Cui Y, et al. Genome-wide association study in a Chinese Han population identifies nine new susceptibility loci for systemic lupus erythematosus. Nat Genet 2009; 41: 1234-1237.

95 Nakano S, Morimoto S, Suzuki S, et al. Up-regulation of the endoplasmic reticulum transmembrane protein UNC93B in the B cells of patients with active systemic lupus erythematosus. Rheumatology 2010; 49: 876-881.

96 Negishi H, Yanai H, Nakajima A, et al. Cross-interference of RLR and TLR signaling pathways modulates antibacterial T cell responses. Nat Immunol 2012; 13: 659-666. 


\section{Nature 2008; 452: 323-328.}

Colina R, Costa-Mattioli M, Dowling RJ, et al. Translational control of the innate immune response through IRF-7.

98 Diebold SS, Kaisho T, Hemmi H, et al. Innate antiviral responses by means of TLR7-mediated recognition of singlestranded RNA. Science 2004; 303: 1529-1531.

99 Lund JM, Alexopoulou L, Sato A, et al. Recognition of single-stranded RNA viruses by Toll-like receptor 7. Proc Natl Acad Sci USA 2004; 101: 5598-5603.

100 Lee HK, Lund JM, Ramanathan B, et al. Autophagy-dependent viral recognition by plasmacytoid dendritic cells. Science 2007; 315: 1398-1401.

101 Tang D, Kang R, Cheh CW, et al. HMGB1 release and redox regulates autophagy and apoptosis in cancer cells. Oncogene 2010; 29: 5299-5310.

102 Deretic V. Autophagy as an innate immunity paradigm: expanding the scope and repertoire of pattern recognition receptors. Curr Opin Immunol 2012; 24: 21-31.

103 Hornung V, Schlender J, Guenthner-Biller M, et al. Replication-dependent potent IFN- $\alpha$ induction in human plasmacytoid dendritic cells by a single-stranded RNA virus. J Immunol 2004; 173: 5935-5943.

104 Guerrero-Plata A, Casola A, Suarez G, et al. Differential response of dendritic cells to human metapneumovirus and respiratory syncytial virus. Am J Respir Cell Mol Biol 2006; 34: 320-329.

105 Guerrero-Plata A, Kolli D, Hong C, et al. Subversion of pulmonary dendritic cell function by paramyxovirus infections. J Immunol 2009; 182: 3072-3083.

106 Schlender J, Hornung V, Finke S, et al. Inhibition of toll-like receptor 7- and 9-mediated alpha/beta interferon production in human plasmacytoid dendritic cells by respiratory syncytial virus and measles virus. J Virol 2005; 79: 5507-5515.

107 Johnson TR, Johnson CN, Corbett KS, et al. Primary human mDC1, mDC2, and pDC dendritic cells are differentially infected and activated by respiratory syncytial virus. PLoS One 2011; 6: e16458.

108 Swiecki M, Gilfillan S, Vermi W, et al. Plasmacytoid dendritic cell ablation impacts early interferon responses and antiviral NK and $\mathrm{CD}^{+} \mathrm{T}$ cell accrual. Immunity 2010; 33: 955-966.

109 Takagi H, Fukaya T, Eizumi K, et al. Plasmacytoid dendritic cells are crucial for the initiation of inflammation and T cell immunity in vivo. Immunity 2011; 35: 958-971.

110 Smit JJ, Rudd BD, Lukacs NW. Plasmacytoid dendritic cells inhibit pulmonary immunopathology and promote clearance of respiratory syncytial virus. J Exp Med 2006; 203: 1153-1159.

111 Wang H, Peters N, Schwarze J. Plasmacytoid dendritic cells limit viral replication, pulmonary inflammation, and airway hyperresponsiveness in respiratory syncytial virus infection. J Immunol 2006; 177: 6263-6270.

112 Lukacs NW, Smit JJ, Mukherjee S, et al. Respiratory virus-induced TLR7 activation controls IL-17-associated increased mucus via IL-23 regulation. J Immunol 2010; 185: 2231-2239.

113 Blasius AL, Giurisato E, Cella M, et al. Bone marrow stromal cell antigen 2 is a specific marker of type I IFNproducing cells in the naive mouse, but a promiscuous cell surface antigen following IFN stimulation. J Immunol 2006; 177: 3260-3265.

114 Davidson S, Kaiko G, Loh Z, et al. Plasmacytoid dendritic cells promote host defense against acute pneumovirus infection via the TLR7-MyD88-dependent signaling pathway. J Immunol 2011; 186: 5938-5948.

115 Holloway JW, Yang IA, Holgate ST. Genetics of allergic disease. J Allergy Clin Immunol 2010; 125: Suppl. 2, S81-S94.

116 Wark PA, Johnston SL, Bucchieri F, et al. Asthmatic bronchial epithelial cells have a deficient innate immune response to infection with rhinovirus. J Exp Med 2005; 201: 937-947.

117 Contoli M, Message SD, Laza-Stanca V, et al. Role of deficient type III interferon- $\lambda$ production in asthma exacerbations. Nat Med 2006; 12: 1023-1026.

118 Lopez-Souza N, Favoreto S, Wong H, et al. In vitro susceptibility to rhinovirus infection is greater for bronchial than for nasal airway epithelial cells in human subjects. J Allergy Clin Immunol 2009; 123: 1384-1390.

119 Bochkov YA, Hanson KM, Keles S, et al. Rhinovirus-induced modulation of gene expression in bronchial epithelial cells from subjects with asthma. Mucosal Immunol 2010; 3: 69-80.

120 Gregorio J, Meller S, Conrad C, et al. Plasmacytoid dendritic cells sense skin injury and promote wound healing through type I interferons. I Exp Med 2010; 207: 2921-2930.

121 Lambrecht BN, Hammad H. The airway epithelium in asthma. Nat Med 2012; 18: 684-692.

122 Chen G, Wan H, Luo F, et al. Foxa2 programs Th2 cell-mediated innate immunity in the developing lung. J Immunol 2010; 184: 6133-6141.

123 Willart MA, Deswarte K, Pouliot P, et al. Interleukin-1 $\alpha$ controls allergic sensitization to inhaled house dust mite via the epithelial release of GM-CSF and IL-33. J Exp Med 2012; 209: 1505-1517.

124 Kay AB, Phipps S, Robinson DS. A role for eosinophils in airway remodelling in asthma. Trends Immunol 2004; 25: 477-482.

125 Allen JE, Wynn TA. Evolution of Th2 immunity: a rapid repair response to tissue destructive pathogens. PLoS Pathog 2011; 7: e1002003.

126 Palm NW, Rosenstein RK, Medzhitov R. Allergic host defences. Nature 2012; 484: 465-472.

127 Lambrecht BN, Hammad H. Lung dendritic cells in respiratory viral infection and asthma: from protection to immunopathology. Annu Rev Immunol 2012; 30: 243-270.

128 Kool M, van Nimwegen M, Willart MA, et al. An anti-inflammatory role for plasmacytoid dendritic cells in allergic airway inflammation. J Immunol 2009; 183: 1074-1082.

129 Lombardi V, Speak AO, Kerzerho J, et al. CD8 $\alpha^{+} \beta^{-}$and CD8 $\alpha^{+} \beta^{+}$plasmacytoid dendritic cells induce Foxp3 regulatory $\mathrm{T}$ cells and prevent the induction of airway hyper-reactivity. Mucosal Immunol 2012; 5: $432-443$.

130 Schlitzer A, Heiseke AF, Einwächter H, et al. Tissue-specific differentiation of a circulating CCR9- pDC-like common dendritic cell precursor. Blood 2012; 119: 6063-6071.

131 Bar-On L, Birnberg T, Lewis KL, et al. CX3CR1 ${ }^{+} \mathrm{CD} 8 \alpha^{+}$dendritic cells are a steady-state population related to plasmacytoid dendritic cells. Proc Natl Acad Sci USA 2010; 107: 14745-14750.

132 Björck P, Leong HX, Engleman EG. Plasmacytoid dendritic cell dichotomy: identification of IFN- $\alpha$ producing cells as a phenotypically and functionally distinct subset. J Immunol 2011; 186: 1477-1485.

133 Yin Z, Dai J, Deng J, et al. Type III IFNs are produced by and stimulate human plasmacytoid dendritic cells. J Immunol 2012; 189: 2735-2745. 